\title{
A CRIANÇA E O ADOLESCENTE COM DIABETES MELLITUS TIPO 1: DESDOBRAR DO CUIDADO FAMILIAR
}

\author{
Tatiana da Silva Melo Malaquias ${ }^{1}$, Camilla Delavalentina Cavalini Marques ${ }^{2}$, Alessandra Cristina de Paula \\ Faria $^{3}$, Jussara Simone Lenzi Pupulim ${ }^{4}$, Sonia Silva Marcon ${ }^{5}$, Ieda Harumi Higarashi ${ }^{6}$
}

\begin{abstract}
RESUMO: Estudo descritivo de abordagem qualitativa cujo objetivo foi apreender como ocorre o cuidado familiar às crianças e adolescentes com diabetes mellitus tipo 1. Foram entrevistados sete familiares no período de abril a junho de 2012. Os dados obtidos por entrevista semiestruturada foram submetidos à análise de conteúdo, modalidade temática. Emergiram duas categorias temáticas, que revelaram as transformações ocorridas na vida destas famílias, destacando as relacionadas à restrição alimentar e à falta de suporte adequado por parte das redes sociais, amigos e demais familiares. Desta forma, vislumbra-se a necessidade de educação continuada não somente junto aos familiares que convivem diariamente com esta criança/adolescente, mas também a todos os profissionais envolvidos na assistência, bem como aos membros da família extensa.
\end{abstract}

DESCRITORES: Diabetes mellitus; Criança; Adolescente; Família.

\section{THE DIVISION OF THE CARE OF THE CHILD OR ADOLESCENT WITH TYPE I DIABETES WITH THE FAMILY}

\begin{abstract}
Descriptive study with a qualitative approach, aiming to understand how the family care for children and adolescents with type I diabetes mellitus takes place. Interviews were held with seven family members in April - June 2012. The data obtained through the semistructured interviews were subjected to content analysis of the thematic modality. Two thematic categories emerged, which revealed the transformations which occur in these families' lives, with emphasis placed on those with dietary restrictions and the lack of appropriate support on the part of social networks, friends and other family members. In this way, one can see the need for continuous education, not only for the family members who live with this child/adolescent on a daily basis, but also for all of those professionals involved in the care, as well as members of the extended family.
\end{abstract}

DESCRIPTORS: Diabetes mellitus; Child; Adolescent; Family.

\section{EL NIÑO Y EL ADOLESCENTE CON DIABETES MELLITUS TIPO 1: DESPLEGAR DEL CUIDADO FAMILIAR}

RESUMEN: Estudio descriptivo con abordaje cualitativo cuyo objetivo fue reflexionar acerca de cómo ocurre el cuidado familiar de niños y adolescentes con diabetes mellitus tipo 1. Siete familiares fueron entrevistados en el periodo de abril a junio de 2012. Los datos fueron obtenidos por entrevista semiestructurada y sometidos al análisis de contenido, modalidad temática. De ellos, resultaron dos categorías temáticas, las cuales mostraron las transformaciones que ocurrieron en la vida de las familias analizadas, destacando aquellas asociadas a la restricción alimentar y a la falta de apoyo adecuado por redes sociales, amigos y otros familiares. Así se vislumbra la necesidad de educación continuada no solamente con los familiares que conviven diariamente con este niño/ adolescente, pero con todos los profesionais que hacen parte de la asistencia y con los miembros de la familia ampliada.

DESCRIPTORES: Diabetes mellitus; Niño; Adolescente; Familia.

${ }^{1}$ Enfermeira. Mestre em Enfermagem. Docente de Enfermagem da Universidade Estadual do Centro-Oeste. Guarapuava, PR, Brasil.

${ }^{2}$ Enfermeira. Mestre em Enfermagem. Hospital Universitário da

Universidade Estadual de Maringá. Maringá, PR, Brasil.

${ }^{3}$ Enfermeira. Especialista em Enfermagem Neonatal. Docente de Enfermagem da Universidade Estadual do Centro-Oeste. Guarapuava, PR, Brasil

${ }^{4}$ Enfermeira. Doutora em Enfermagem. Docente de Enfermagem da Universidade Estadual de Maringá. Maringá, PR, Brasil.

${ }^{5}$ Enfermeira. Doutora em Filosofia. Docente de Enfermagem da Universidade Estadual de Maringá. Maringá, PR, Brasil.

${ }^{6}$ Enfermeira. Doutora em Educação. Docente de Enfermagem da Universidade Estadual de Maringá. Maringá, PR, Brasil.

R. Conselheiro Jesuino Marcondes, 520 - 85015-390 - Guarapuava, PR, Brasil

E-mail: tatieangel@yahoo.com.br 


\section{- INTRODUÇÃO}

O diabetes mellitus tipo 1 (DM1), conhecido como diabetes juvenil e/ou insulino dependente, é responsável por cerca de $10 \%$ dos casos de diabetes no Brasil. Neste tipo de diabetes, ocorre a destruição da célula beta do pâncreas, devido a um processo autoimune ou de outras doenças degenerativas que se associam ao DM1, comprometendo a capacidade total de produção de insulina que é necessária para baixar os níveis glicêmicos e assim prevenir a cetoacidose, coma e a morte ${ }^{(1)}$.

Apesar da gravidade do DM1, não existem medidas de prevenção para evitar o seu surgimento, por esta razão, sua incidência vem crescendo ao longo dos anos. No Reino Unido, por exemplo, o DM1 é a terceira doença crônica mais comum na infância, a incidência dobrou nos últimos 20 anos e anualmente surgem 13.5 casos novos em cada 100.000 crianças $^{(2)}$.

É notório o impacto da doença na sociedade devido ao número crescente de pessoas acometidas, mortes prematuras, presença de incapacitação e complicações decorrentes da doença e dos elevados custos envolvidos em seu controle e tratamento ${ }^{(3)}$.

Um estudo realizado na Escócia mostrou que existe estreita relação entre a estrutura familiar e o controle glicêmico no decorrer da infância, sendo que influências psicossociais e educacionais desempenham papel fundamental na determinação dos resultados de gestão do DM1 em crianças. A adolescência também desempenha um importante papel nesta gestão devido às mudanças físicas rápidas da puberdade, as quais podem desencadear relativa resistência aos efeitos da insulina e simultâneas mudanças de desenvolvimento, bem como elevar o nível de estresse mediante pressões dos colegas ${ }^{(2)}$.

No Brasil, um estudo constatou que a criança/adolescente com DM1, quando percebe a dificuldade dos pais em lidarem com sua doença, tende a se isolar do seu meio social, pois esta percepção faz com que elas se sintam diferentes de seus pares ${ }^{(1)}$.

Contudo, é possível inferir o quão dificíl é para os pais a convivência com esta situação de doença, marcada muitas vezes pela sensação de impotência, pela demanda por cuidados novos e específicos, para os quais nem sempre se sentem aptos a atendê-los. Todas estas questões despertam nos famíliares a necessidade de aprender a lidar com sentimentos, sintomas e procedimentos terapêuticos desconhecidos até então, além de terem que aprender também a controlar a doença ${ }^{(3)}$.

Uma reação bastante comum nestes casos é a autopunição paterna, o que faz com que estes assumam uma postura protecionista, tornando a criança/adolescente dependente de seus cuidados, bem como em pólo centralizador das atenções, apreensões e angústias da família ${ }^{(2,4)}$. Neste sentindo, vislumbra-se a necessidade de assistência diferenciada por parte dos profissionais de saúde, os quais poderão direcionar sua atuação para o cuidado focalizado na família e na criança/adolescente com doença crônica ${ }^{(1)}$.

Assim, ratifica-se a importância deste estudo, uma vez que busca evidenciar lacunas destacadas pelos principais cuidadores das crianças e adolescentes com DM1, de modo a subsidiar a atuação dos profissionais de saúde na inplementação de estratégias que possam melhor capacitar estes cuidadores. Diante do exposto, definiu-se como objetivo do estudo apreender como ocorre o cuidado familiar às crianças e adolescentes com diabetes mellitus tipo 1.

\section{METODOLOGIA}

Trata-se de pesquisa exploratória e descritiva, realizada no município de Paranavaí - PR, no âmbito das Unidades Básicas de Saúde (UBS). Este município tinha em 2010 uma população de 81.590 habitantes ${ }^{(5)}$, dos quais 24.308 eram crianças e adolescentes. Na área da saúde, o município possuía 16 equipes da Estratégia Saúde da Família (ESF), distribuídas em 15 UBS.

Os colaboradores do estudo foram os familiares das crianças e adolescentes com DM1 com idade igual ou superior a 18 anos e cuja criança/adolescente acometida pela doença apresentasse idade igual 
ou superior a um ano de idade. Os dados foram coletados nos domicílios das famílias, entre os meses de abril e junho de 2012, por meio de entrevista semiestruturada. O instrumento utilizado na entrevista foi um roteiro elaborado pelas autoras, constituído de duas partes: a primeira abordava aspectos relacionados à caracterização sociodemográfica dos sujeitos e a segunda um roteiro semiestruturado com perguntas baseadas no objetivo do estudo.

Realizou-se um levantamento nas 16 equipes de ESF do município junto à equipe de enfermagem e agentes comunitários de saúde (ACS), localizando-se 11 famílias com crianças/adolescentes acometidos pelo DM1, sendo que destas, apenas sete famílias aceitaram participar do estudo perante o convite, que se deu por meio de visita domiciliar.

Os colaboradores foram selecionados segundo os seguintes critérios: ser cuidador principal da criança/adolescente com DM1; tempo de diagnóstico da doença do filho maior que um ano. Para garantir o anonimato, os familiares foram identificados com a letra $\mathrm{P}$ e numeração arábica de 1 a 7 , de acordo com a ordem das entrevistas. No que se refere às crianças/adolescentes filhos dos participantes do estudo, foram identificados pela letra $\mathrm{F}$ e numeração arábica de 1 a 7 , referenciando de qual familiar ele é filho (F1 - filho do familiar 1).

Para a interpretação dos dados, as entrevistas foram transcritas na íntegra e submetidas a um processo de análise de conteúdo, modalidade temática ${ }^{(6)}$, respeitando suas etapas: a primeira foi a organização das informações, utilizando procedimentos de leitura flutuante, hipóteses, objetivos e elaboração de indicadores para fundamentar a interpretação; a segunda de codificação dos dados, através das unidades de registro; e a última etapa de categorização, quando os elementos são classificados de acordo com semelhanças e diferenciações, sendo reagrupados por características comuns. Emergiram duas categorias temáticas: Mudança de hábitos alimentares: a família convivendo com o diabetes e Controle alimentar: vigilância constante da criança/adolescente.

O projeto foi aprovado pelo Comitê Permanente de Ética de Pesquisa com Seres Humanos da Universidade Estadual de Maringá (parecer $n^{\circ}$ 16829) e seguiu os preceitos da Resolução 466/2012 ${ }^{(7)}$ do Conselho Nacional de Saúde para pesquisas com seres humanos.

\section{RESULTADOS}

A pesquisa teve como participantes sete familiares de crianças/adolescente com diabetes mellitus tipo 1, sendo seis mães e um pai conforme pode ser verificado na Tabela 1. Em relação aos filhos dos colaboradores, a maioria deles era adolescente e com diagnóstico da doença há mais de três anos (Tabela 2). As categorias temáticas do estudo são apresentadas na sequência.

\section{Mudança de hábitos alimentares: a família convivendo com o diabetes}

Uma das alterações provocadas pelo DM1 que mais se destaca é representada pela alimentação e se relaciona com a disfunção fisiológica do metabolismo de carboidratos. Tais mudanças foram apontadas pelos informantes do estudo, ao relatarem que o principal modo de lidar com a doença foi a implementação de modificações nos hábitos alimentares da criança/adolescente e mais ainda, a tentativa de expandir estas mudanças para os demais membros da família.

[...] Não tem doce dentro da minha casa! É muita fruta, muita verdura... [apontando para a comida que estava sendo preparada] Saudável! Peito de frango que eu estou cozinhando. (P1)

Mudou bastante, mudou a alimentação. A gente já cuidava um pouco, mas não era tanto, a parte de frituras a gente diminuiu bastante, principalmente os doces... (P2)

Teve mudança na alimentação da gente, por causa dele né?... Comer pouca coisa, não muito doce, diminuir o açúcar, adoçante pra ele. (P4)

Doce, massa... eu fazia bastante. Agora diminui, fiz mudança dos pães de massa branca para integral. (P7)

Desta forma, alguns alimentos que não faziam parte do cardápio da família, passaram a ser inseridos, enquanto outros foram adaptados ou excluídos. 
Tabela 1 - Características socioeconômicas dos familiares de crianças/adolescentes com diabetes mellitus tipo 1. Paranavaí, PR, Brasil, 2012

\begin{tabular}{llcll} 
Código & Gênero/Parentesco & Idade & Escolaridade & Renda Mensal Familiar \\
\hline P1 & Feminino (mãe) & 29 & Ensino médio incompleto & 1 salário mínimo \\
\hline P2 & Feminino (mãe) & 55 & Ensino superior & Maior que 3 salários mínimos \\
\hline P3 & Feminino (mãe) & 63 & Ensino fundamental incompleto & 3 salários mínimos \\
\hline P4 & Feminino (mãe) & 36 & Ensino médio completo & 3 salários mínimos \\
\hline P5 & Feminino (mãe) & 43 & Ensino médio completo & 3 salários mínimos \\
\hline P6 & Feminino (mãe) & 32 & Ensino médio completo & Maior que 3 salários mínimos \\
\hline P7 & Masculino (pai) & 45 & Ensino médio completo & 3 salários mínimos
\end{tabular}

Tabela 2 - Características das crianças/adolescentes com diabetes mellitus tipo 1. Paranavaí, PR, Brasil, 2012

\begin{tabular}{llcc} 
Código & Gênero & Idade (anos) & Tempo de Diagnóstico do DM1 (em anos) \\
\hline F1 & Masculino & 8 & 3 \\
\hline F2 & Feminino & 14 & 8 \\
\hline F3 & Masculino & 12 & 3 \\
\hline F4 & Feminino & 9 & 6 \\
\hline F5 & Masculino & 15 & 15 \\
\hline F6 & Feminino & 12 & 2 \\
\hline F7 & Masculino & 18 & 1
\end{tabular}

\section{Controle alimentar: vigilância alimentar da criança/adolescente}

É comum observar-se, entre os familiares, posturas de preocupação em relação ao controle alimentar da criança/adolescente, tanto no âmbito doméstico, como em outros cenários de convivência social destas famílias. Ademais, a adoção de uma vigilância constante ou mesmo as imposições de regras rígidas refletem a tentativa destes cuidadores de evitar, a qualquer custo, as repercussões danosas do desequilíbrio glicêmico em suas crianças.

Às vezes me desespero na parte de alimentação... porque é o que mais precisa ser controlado e preciso da ajuda da família e não tenho apoio. Eles [familiares] comem doces perto dela [adolescente] e ela fica com vontade, mas não se importam com isso... (P2)

Na escola, a gente tem que controlar a comida dele! Não pode dar qualquer coisa. Nós conversamos com a professora, ela sabe de tudo. A dificuldade é não o deixar comer tudo que tem vontade. (P3)

Tem que ficar cuidando o tempo todo e, mesmo assim, às vezes um descuido e a diabetes aumenta e tem que internar. A gente se preocupa com tudo porque é diferente. É uma menininha fraca que tem que tratar completamente diferente dos outros. Ela é bem mais delicada. (P5)

Com este propósito, os familiares passam a exercer um papel de vigília sobre aquilo que os filhos ingerem e impõem regras, não só no âmbito doméstico, mas em todo o contexto social.

\section{- DISCUSSÃO}

A American Diabetes Association ${ }^{(8)}$ destaca como principais objetivos da Terapia Médico Nutricional ao paciente com DM1: a) prevenir complicações crônicas por meio da correta ingesta nutricional acompanhada de alterações no estilo de vida; b) manter o prazer de se alimentar, limitando apenas determinados alimentos (conforme evidências científicas); c) satisfazer as necessidades nutricionais individuais, de acordo com aspectos culturais e pessoais; d) motivar o paciente para a regulação de mudanças; e)integrar o regime de terapia insulínica ao estilo de vida do paciente. 
O impacto pela mudança alimentar no cenário familiar vem em decorrência das necessidades ou demandas para controle da doença, já que os estudos apontam que a terapia nutricional é parte imprescindível no tratamento do diabetes mellitus ${ }^{(8-9)}$. Entretanto, a reestruturação alimentar é uma das mudanças mais difíceis que a família tem que enfrentar no convívio com diabetes, por acarretar restrições e limitações até então desnecessárias ${ }^{(10)}$.

É preciso ter em mente que a mudança de hábitos, seja alimentar ou de outra natureza, envolve mais do que simplesmente deixar de realizar algo ou passar a realizá-lo de maneira diferente. Tais mudanças refletem diretamente na socialização do indivíduo como ficou evidente em um estudo(11) no qual as autoras referem que as crianças com DM1 acreditam que todos os cuidados que devem ser tomados com relação à doença, interferem de maneira direta na sua vida social. Isto faz com que as mesmas se sintam não apenas diferentes das outras crianças, mas também, privadas de um tempo precioso de suas vidas, para destiná-lo aos inúmeros cuidados no controle da doença, em detrimento de outras atividades corriqueiras e mais prazerosas.

Neste aspecto, destaca-se a possibilidade de ocorrerem dificuldades de compreensão e/ou aceitação por parte da criança/adolescente, em função da necessidade de "abrir mão" de certos prazeres e do cotidiano comum a outras crianças da mesma idade. O papel dos pais ou responsáveis, neste cenário de transição, torna-se elemento fundamental para o sucesso terapêutico e a garantia de padrões futuros de qualidade de vida dos pequenos pacientes ${ }^{(12-13)}$.

Caracterizada como uma doença infanto-juvenil, o DM1 emerge, na maioria dos casos, numa fase da vida em que o indivíduo ainda não possui competências suficientes para o seu autocuidado. De fato, na maior parte dos casos, a doença surge em crianças numa faixa etária na qual habilidades cognitivas e a maturação emocional ainda não se encontram suficientemente organizadas para o planejamento ou para a tomada de decisões em relação a seu regime terapêutico ${ }^{(11)}$.

Os relatos permitem evidenciar que, embora o diagnóstico, na maioria dos casos, tenha ocorrido há mais de três anos, os familiares ainda apresentam dificuldades em seu cotidiano com uma criança/ adolescente com DM1. Neste cenário, as demandas relativas ao controle rigoroso da alimentação têm repercussões não apenas no processo terapêutico, mas traz implicações para todo o processo de socialização da criança e de sua família.

Há assim, uma expectativa dos pais em relação ao comportamento dos demais familiares, no sentido de uma corresponsabilização com relação ao controle alimentar da criança diabética, e de modo a evitar frustrações da criança frente à privação alimentar.

Lidar com o desejo de comer e com a culpa por ter comido o que não deveria é umas das dificuldades no processo de convivência com a doença. Estes aspectos são percebidos principalmente nos ambientes de convívio social da criança, como na escola, onde a mesma se vê obrigada a levar seu lanche de casa, o que restringe sua participação nas refeições em conjunto e, consequentemente, dificulta o processo de interação social neste ambiente ${ }^{(4)}$.

Ao mesmo tempo, sabe-se que um fator intrínseco para que haja o desenvolvimento do autocuidado na criança é o envolvimento dos amigos, dos pais e da família estendida no processo terapêutico da doença. E este envolvimento promove o aumento da autoconfiança da criança e também o entendimento acerca da importância da adesão ao tratamento correto ${ }^{(12)}$.

Para a criança/adolescente com DM1, o manejo da doença relaciona-se diretamente com o quanto ela compreende de seu estado de saúde, bem como com outros aspectos importantes tais como: o autocuidado, o cotidiano escolar, o apoio dos amigos, da família e dos profissionais da saúde. No que concerne ao papel dos profissionais, há que se destacar a importância da atuação do enfermeiro, que deve estar atento para a avaliação desses aspectos, para identificá-los, e então planejar e implementar estratégias de intervenção que facilitem o cuidado para toda a família e para a criança/adolescente ${ }^{(1,12)}$.

Para tanto, o profissional de enfermagem deve envolver a família no cuidado da criança/adolescente com DM1, de modo a capacitar seus integrantes para enfrentar as limitações e desafios impostos pela doença. Também se faz necessário estimular a autonomia das crianças/adolescentes e familiares, de modo que estes possam atuar como multiplicadores de informações, auxiliando outras pessoas que necessitem de apoio no tratamento do diabetes ${ }^{(9)}$. 


\section{- CONCLUSÃO}

O estudo possibilitou compreender o quanto o diabetes mellitus tipo 1 altera significativamente o modo de vida do doente e de sua família, principalmente em relação aos aspectos alimentares. A mudança de hábitos alimentares em decorrência do DM1 traz modificações importantes no cotidiano familiar, social e econômico dos participantes, além de preocupações com o seguimento da dieta adequada em ambientes fora do contexto familiar pelos filhos, como por exemplo, a escola, onde os pais não conseguem exercer uma vigilância constante e efetiva sobre aquilo que os filhos realmente estão ingerindo.

Os pais relataram que as dificuldades em se manter uma alimentação adequada para as crianças e adolescentes com diabetes, pode interferir na capacidade dos pacientes em aderir e controlar a doença, pois eles não possuem maturidade emocional e cognitiva plenamente desenvolvidas, para o autocuidado efetivo.

Deste modo, emerge a necessidade de uma abordagem familiar individualizada por parte da equipe multidisciplinar de saúde, permitindo que os mesmos expressem seus anseios e conflitos ao lidar com o DM1, de modo a planejar uma assistência no qual a família seja agente ativo no manejo do cuidado à doença. Por outro lado, a boa relação entre criança, família e profissionais de saúde facilita o tratamento e controle da doença, além de estabelecer laços de confiança e respeito entre os familiares e a equipe de saúde.

\section{REFERÊNCIAS}

1. Leal DT, Fialho FA, Dias IMAV, Nascimento L, Arruada WC. Diabetes na infância e adolescência: o enfrentamento da doença no cotidiano da família. HU Rev. 2009; (35)4:288-95.

2. Mcnamara R, Robling M, Hood K, Bennert K, Chanon S, Cohen D, et al. Development and evaluation of a psychosocial intervention for children and teenagers experiencing diabetes (DEPICTED): a protocol for a cluster randomised controlled trial of the effectiveness of a communication skills training programme for healthcare professionals working with young people with type 1 diabetes. BMC Health Serv. Res. 2010; 10(36):2-10.

3. de Souza IVB, Marques DKA, Lacerda ORM, Collet N. Percepção das mães frente ao diagnóstico do filho com diabetes mellitus tipo 1. Cogitare enferm. 2011; 16(1):43-8.

4. Leal DT, Fialho FA, Dias IMAV, Nascimento L, Arruada WC. A vivência dos familiares de crianças e adolescentes portadores de diabetes mellitus tipo 1. Rev. Eletr. Enf. [Internet] 2012; 14(1) [acesso em 24 jun 2015]. Disponível: http://www.revistas.ufg.br/index.php/fen/article/viewFile/9641/15572

5. Instituto Brasileiro de Geografia e Estatítisca (IBGE). Cidades@ [Internet] [acesso em 19 mar 2012]. Disponível: http://www.cidades.ibge.gov.br/xtras/home.php

6. Bardin L. Análise de conteúdo. Lisboa: Edições 70; 2011.

7. Ministério da Saúde (BR). Conselho Nacional de Saúde. Diretrizes e normas regulamentadoras de pesquisa envolvendo seres humanos. Resolução n. 466, de 12 de dezembro de 2012. Brasília; 2012.

8. American Diabetes Association. Nutrition Recommendations and interventions for diabetes. Diabetes Care. [Internet] 2008; 31(Suppl.1) [acesso em 21 jul 2012]. Disponível: http://care.diabetesjournals.org/content/31/ Supplement_1/S61.short

9. Queiroz KC, Silva IN, Alfenas RCG. Associação entre fatores nutricionais e o controle glicêmico de crianças e adolescentes com diabetes melito tipo 1. Arq Bras Endocrinol Metab. [Internet] 2010; 54(3) [acesso em 24 jul 2015]. Disponível: http://dx.doi.org/10.1590/S0004-27302010000300011

10. Martins EMCS, de Ataíde MBC, de Silva DMA, Frota MA. Vivência de mães no cuidado a criança diabética tipo 1. Rev Rene [Internet] 2013; 14(1) [acesso em 24 jul 2015]. Disponível: http://www.revistarene.ufc.br/revista/index. php/revista/article/viewFile/1332/pdf 
11. Sparapani VC, Borges ALV, Dantas IRO, Pan R, Nascimento LC. A criança com Diabetes Mellitus Tipo 1 e seus amigos: a influência dessa interação no manejo da doença. Rev. Latino-Am. Enfermagem. [Internet] 2012; 20(1) [acesso em 30 jul 2012]. Disponível: http://dx.doi.org/10.1590/S0104-11692012000100016

12. Nascimento LC, Amaral MJ, Saparani VC, Fonseca LMM, Nunes MDRN, Dupas G. Diabetes mellitus tipo 1: evidências da literatura para seu manejo adequado, na perspectiva de crianças. Rev. esc. Enferm. USP. [Internet] 2011; 45(3) [acesso em 25 ago 2012]. Disponível: http://dx.doi.org/10.1590/S0080-62342011000300031

13. Araújo YB, Collet N, Gomes IP, Amador DD. Saberes e experiências de adolescentes hospitalizados com doença crônica. Rev. enferm. UERJ. [Internet] 2011; 19(2) [acesso em 30 nov 12]. Disponível: http://www.facenf. uerj.br/v19n2/v19n2a17.pdf 